\title{
Aprovechamiento sustentable del recurso hídrico mediante un sistema de captación de agua de lluvia y red de suministro
}

\section{Sustainable use of water resources through a rainwater harvesting system and supply network}

\author{
CASTILlO-TÉLLEZ, Margarita†*’, CASTILLO-TÉLLEZ, Beatriz”, MEJÍA-PÉREZ, Gerardo \\ Alberto" y HERNÁNDEZ-CRUZ, Luz María’
}

Universidad Autónoma de Campeche, Facultad de Ingeniería, Campus V, predio s/n por Av Humberto Lanz Cárdenas y Unidad Habitacional Ecológica ambiental, Col. Ex Hacienda Kalá, C.P. 24085, San Francisco de Campeche, Campeche, México.

"Centro Universitario del Norte, Universidad de Guadalajara. Km. 191, México 45D No. 23, $46200 \mathrm{Jal}^{2}$

ID $1^{\text {er }}$ Autor: Margarita, Castillo-Téllez, ORC ID: 0000-0001-9639-1736, Researcher ID Thomson: S-2283-2018, CVU CONACYT ID: 210428

ID $1^{\text {er }}$ Coautor: Beatriz, Castillo-Téllez / ORC ID: 0000-0003-3747-6320, Researcher ID Thomson: S-2264-2018, CVU CONACYT ID: 210564

ID $2^{\text {do }}$ Coautor: Gerardo Alberto, Mejía-Pérez / ORC ID: 0000-0003-1701-1171

ID $3^{\text {er }}$ Coautor: Luz María, Hernández-Cruz / ORC ID: 0000-0002-0469-5298, Researcher ID Thomson: H-3153-2018, CVU CONACYT ID: 662220

DOI: $10.35429 /$ JOIE.2020.14.4.27.34

Recibido Abril 25, 2020; Aceptado Junio 30, 2020

\begin{abstract}
Resumen
La necesidad energética actual permite que se apliquen nuevas tecnologías y métodos para satisfacer las necesidades de una población a fin de contaminar lo menos posible y hacer que los sistemas sean más eficientes. Este trabajo presenta el diseño e instalación de un sistema de recolección de agua de lluvia en la Facultad de Ingeniería de la Universidad Autónoma de Campeche y su utilización en un jardín conformado por un cuerpo de agua y riego de hortalizas, incluyendo la implementación de energías renovables, demostrando que las tecnologías sustentables aplicadas de una manera correcta pueden estar en armonía con el medio ambiente. El potencial hídrico de una caída libre es utilizable para la generación de energía eléctrica limpia mediante una microturbina hidráulica, capaz de alimentar una bomba sumergible que permite la recirculación de agua dentro del sistema. El proyecto se enfoca en la agricultura, acuacultura e hidráulica que enlaza la sustentabilidad que podría alcanzar con la aplicación de las tecnologías renovables.
\end{abstract}

Potencial hídrico, Captación de agua de lluvia, Energías renovables

\begin{abstract}
The current energy need allows new technologies and methods to be applied to meet the needs of a population to pollute as little as possible and make systems more efficient. This work presents the design and installation of a rainwater harvesting system in the Faculty of Engineering of the Autonomous University of Campeche and its use in a garden made up of a body of water and vegetable irrigation, including the implementation of renewable energy, demonstrating that sustainable technologies applied correctly can be in harmony with the environment. The water potential of a free fall is usable for the generation of clean electrical energy using a hydraulic micro-turbine, capable of supplying a submersible pump that allows the recirculation of water within the system. The project focuses on agriculture, aquaculture and hydraulics that link the sustainability that could be achieved thanks to the application of renewable technologies.
\end{abstract}

Water potencial, Rainwater harvesting, Renewable energies

Citación: CASTILLO-TÉLLEZ, Margarita, CASTILLO-TÉLlEZ, Beatriz, MEJÍA-PÉREZ, Gerardo Alberto y HERNÁNDEZ-CRUZ, Luz María. Aprovechamiento sustentable del recurso hídrico mediante un sistema de captación de agua de lluvia y red de suministro. Revista de Ingeniería Innovativa. 2020. 4-14:27-34.

\footnotetext{
*Correspondencia al Autor (Correo Electrónico: mcastill@uacam.mx)

$\dagger$ Investigador contribuyendo como primer autor.
} 


\section{Introducción}

El agua es un recurso vital para la reproducción vegetal y animal. Bajo la perspectiva del calentamiento global, el problema de la escasez de agua tiende a empeorar en aquellas regiones en las que ya se presenta déficit, ya sea por la tendencia de reducción de los niveles de precipitación o por el aumento de los niveles de evaporación y transpiración. De acuerdo con la SEMARNAT y la Comisión Nacional del Agua, en México llueve aproximadamente $711 \mathrm{~mm}$ de lluvia al año ( $1 \mathrm{~mm}$ de lluvia es igual a $\left.1 \mathrm{l} / \mathrm{m}^{2}\right)$. Sin embargo, el agua pluvial cae de manera desigual a lo largo de todo el territorio del país y el $67 \%$ de las lluvias se concentra entre los meses de junio y septiembre. En Campeche se tienen lluvias prominentes en tres meses en específico: julio, agosto y septiembre. Sin embargo, en los 9 meses restantes se tiene carencia de precipitaciones, la lluvia promedio acumulada mensual es de $5.9 \mathrm{~mm}$ que representa el $24.8 \%$ de la lluvia promedio histórica del mes, la cual es de 23.9 mm (CONAGUA, 2018).

De los 6700 millones de habitantes del planeta, el $21 \%$ (1 400 millones) no tienen acceso al agua entubada. En el tercer mundo, 85 $\%$ de las enfermedades de la población se derivan de la mala calidad del agua que se consume, la cual provoca la pérdida de más de 5,000 vidas diariamente. Cada año se pierden 443 millones de días escolares por enfermedades relacionadas con el agua. Millones de mujeres dedican hasta cuatro horas diarias al acopio de agua (Anaya, 2011).

Existe una amplia gama de técnicas y tecnologías para mejorar la forma en la que se utiliza el agua, tanto la potable como las aguas grises y negras. Algunas sólo ayudan a ahorrar agua, como los difusores o inodoros de bajo consumo, otras tratan de hacer reutilizables las aguas de desecho o permiten captar agua de lluvia (Robles, Näslund-hadley, Ramos, \& Paredes, 2015).

La captación y el aprovechamiento de la lluvia representan sólo una de las estrategias en el uso racional del agua. La mayoría de las técnicas de captación de lluvia tienen un origen empírico y han sido desarrolladas a lo largo del tiempo, a partir de las civilizaciones ancestrales de Meso y Sudamérica y de otras regiones del mundo.
En los últimos 30 años, se han perfeccionado muchas técnicas gracias al aporte de diferentes instituciones y países (En \& Nivel, 2000). Hay una gran variedad de técnicas adaptadas a diferentes situaciones, mismas que cumplen diferentes finalidades (FAO, 2013).

Debido a que el incremento de la demanda de agua está creciendo de forma exponencial y la oferta en algunos lugares del mundo, incluso en nuestro país, no alcanza a ser suficiente para suplir necesidades básicas, resulta entonces una alternativa interesante el aprovechamiento de aguas lluvias, lo cual redunda en menores costos operativos para las edificaciones y un aporte para la sociedad en términos ecológicos (López, Palacios, Anaya, Chávez, \& Enrique, 2017).

En este trabajo se presenta el diseño, construcción e implementación de un sistema de captación de agua de lluvia, en la Facultad de Ingeniería de la Universidad Autónoma de Campeche, conectado al suministro de red hidráulica, el cual consta de diferentes componentes como son, filtración de agua de lluvia para eliminar impurezas, aprovechamiento del riego del jardín y hortalizas para fomentar la tecnología de secado solar dirigido a la agroindustria sustentable, generación de energía eléctrica limpia mediante una microturbina hidráulica y un sistema fotovoltaico, purificación de agua por medio de destiladores solares y mantenimiento de vida acuática mediante un estanque artificial.

\section{Descripción general del sistema}

El diseño del sistema para aprovechamiento del recurso hídrico y captación del mismo, busca aparte de la utilización del agua almacenada, aplicaciones para sistemas de riego, (las hortalizas se utilizan para estudios en el Laboratorio de Secado Solar de Alimentos ubicado en la Facultad de Ingeniería), generación de energía eléctrica, conservación de vida acuática y procesos de purificación de agua mediante energía solar; se buscó que tuviera un impacto visual atractivo para todo aquel que estudie, trabaje o visite la facultad de ingeniería. Este sistema está basado en el aprovechamiento del potencial energético del agua, en un espacio con una altura considerable, con la finalidad de mover un generador, evitando el uso de un sistema de bombeo de energía de la red. 
Un parámetro importante es la purificación del agua recolectada, la tecnología de destilación solar permite purificarla, aunado a pruebas necesarias para corroborar la calidad de agua destilada obtenida para su posterior uso en limpieza de utensilios en laboratorios de la misma facultad. Los mantenimientos requeridos son mínimos por los tipos de materiales utilizados, mediante el seguimiento de una calendarización se realizan la limpieza de filtros, inspección de tuberías, etc. Al estar conectado el sistema de captación a la red de suministro de agua potable, se garantiza la obtención de agua todo el año.

\section{Materiales y Métodos}

\section{- $\quad$ Selección de una turbina}

La operación de arranque de los distintos tipos de uso en la actualidad difiere según la forma de energía usada para conducirlos. Los rangos de trabajo están delimitados por el caudal (Q) y la altura $(\mathrm{H})$ que se utilizarán y que a su vez arrojara una potencia $(\mathrm{P})$ obtenida en la turbina (Figura 1).

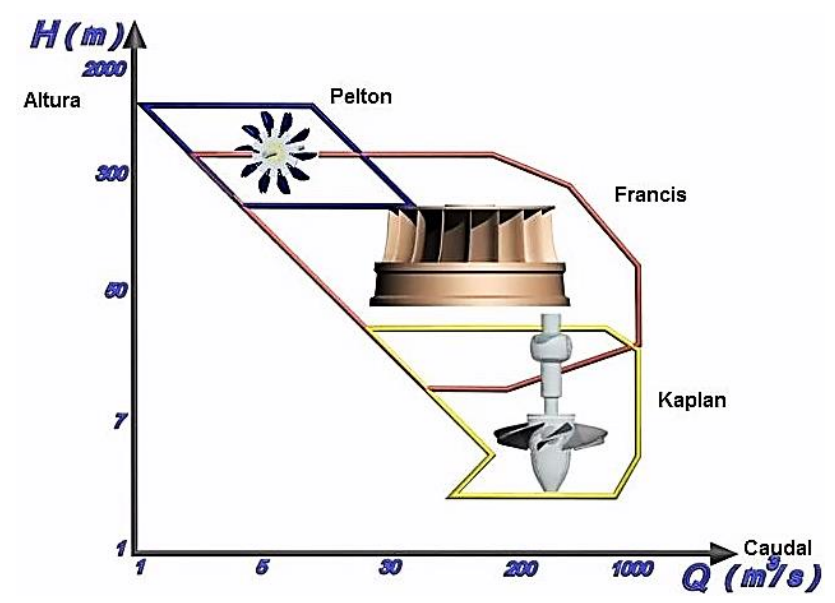

Figura 1 Selección de una turbina

Fuente:https://www.areatecnologia.com/mecanismos/tur binas-hidraulicas.html

\section{- $\quad$ Selección de una bomba}

Para la selección del modelo adecuado de bomba se recurrió a las curvas características de la bomba, aportadas por el fabricante de esta. Se necesitan tres curvas diferentes: las de caudal y carga de la bomba, llamadas curvas $\mathrm{QH}$; las de potencia del motor necesario $(\mathrm{kW})$ y las de Carga Positiva Neta de Aspiración Requerida (NPSHr).

\section{- Sistema de recolección de agua de lluvia}

Para fines potables, únicamente la tubería de PVC es la adecuada, ya que sólo este tipo de tubería es fabricada con materiales vírgenes o no reciclados que pudieron haber obtenido contaminantes de usos previos.

Para evitar la transportación de hojas y basura desde una azotea, es importante tomar en cuenta la colocación de una rejilla en un marco metálico que cubra todo el canal que recolectará el agua pluvial. Las rejillas son importantes ya que reducen la frecuencia en el mantenimiento, eliminan materiales inflamables de la azotea y reducen ambientes aptos para la formación de mosquitos.

\section{- $\quad$ Sistema de distribución de agua}

Es el sistema que lleva el agua desde la zona de almacenaje hasta donde va a ser utilizada. El agua almacenada es distribuida por medio de sistemas de bombeo, debido a la presión requerida. Estas bombas tienen válvulas que regulan los niveles de agua requeridos. Para abastecer un sistema de riego es necesario colocar un filtro junto con la bomba utilizada para evitar que las tuberías para riego se tapen.

\section{- Almacenamiento}

El sobre-flujo del tanque debe desalojar el exceso de agua. La salida de este componente conviene que sea recibido por un área previamente preparada al exterior, o bien a un sistema de drenaje. La salida de agua del tanque lleva al sistema de distribución. El sistema de sobre-flujo tiene que ser colocado para aprovechar el máximo volumen de agua ("Guía De Diseño Para Captación Del Agua De Lluvia," 2004). En este caso se contempló un sello de aire de 14 pulgadas el cual es necesario entre la superficie del agua y la entrada de agua alternativa. Una válvula "check" fue colocada para evitar contaminación adicional desde el sistema alterno.
CASTILLO-TÉLlEZ, Margarita， CASTILLO-TÉLlEZ, Beatriz, MEJÍA-PÉREZ, Gerardo Alberto y HERNÁNDEZCRUZ, Luz María. Aprovechamiento sustentable del recurso hídrico mediante un sistema de captación de agua de lluvia y red de suministro. Revista de Ingeniería Innovativa. 2020 
Parte del agua recolectada se utilizará para otras actividades por lo que, si se diera el caso de requerir de cierta presión para llegar a su destino, se contempló usar una bomba sumergible que permitiera un flujo continuo, también ubicar el tanque de almacenamiento a una altura específica $\mathrm{y}$, por consiguiente, también se tuvo que diseñar las tuberías para alcanzar la generación adecuada de la turbina hidráulica para la alimentación de dicha bomba sumergible.

Para la captación del agua de lluvia en un sistema combinado como es el caso, se requiere realizar una "T" en el tubo posterior al tanque de almacenamiento superior. Dicha tubería tendrá una válvula check (no retorno), para que en el momento de que llueva y se inunde el nivel de la azotea ingrese agua al sistema. Para la época de lluvia se mantiene cerrada la válvula que suministra agua de la red al sistema. Los filtros de agua de lluvia están ubicados de tal manera que faciliten su mantenimiento.

El agua recolectada viaja por un sistema de tuberías hasta el suelo. Con la energía potencial moverá los álabes de una microturbina hidráulica que generará la suficiente energía para alimentar una bomba y el excedente en iluminación artificial, esto con el fin de obtener el atractivo visual que se busca. A la salida de la turbina la tubería PVC transportará el agua hacia el estanque teniendo una salida adicional, la cual está destinada a riego de plantas que podrían representar un potencial en la agricultura.

\section{Materiales y herramientas}

- $\quad$ Dos tanques de almacenamiento de 11001.

- 1 bombas sumergibles solares.

- Tubería PVC de 1", 2" y 4".

- $\quad$ Codos $90^{\circ}$.

- $\quad$ Resistol para PVC hidráulico.

- Cemento.

- Block.

- Turbina hidráulica Pelton.

- Conductores eléctricos.

- Cinta aislante.

- Destilador solar.

- Pala.

- Pico.

- Pinzas eléctricas.

- Desarmadores.

- Segueta.

- Martillo.

- Cuchara de albañil

\section{Resultados y discusión}

\section{Dimensionamiento integral del sistema}

El dimensionamiento del proyecto dependió del espacio que se tiene disponible para ubicar el sistema de suministro y captación donde se almacena el agua, esto limita la capacidad tanto del sistema de destilación que se buscó implementar, como del área del estanque y jardín que se busca alimentar con el agua captada. Los parámetros de diseño son:

\section{a) Diseño de turbina}

$$
Q=\frac{p}{p g h m}=\frac{1 \mathrm{~kW}}{\left(1000 \frac{\mathrm{kg}}{\mathrm{m}^{3}}\right)\left(9.81 \frac{\mathrm{m}}{\mathrm{s}^{2}}\right)(12 \mathrm{~m})(0.95)}=0.0089 \frac{\mathrm{m}^{3}}{\mathrm{~s}}=8.94 \frac{\mathrm{l}}{\mathrm{s}}
$$

\section{b) Velocidad del cause}

$$
C 1=\sqrt{1-\frac{\Delta H i}{H}} 2 g H \rightarrow C 1=k c \sqrt{2 g H}
$$

Se considera $\mathrm{kc}=0.97$

$$
C 1=0.97 \sqrt{2\left(9.81 \frac{m}{s^{2}}\right)(12 m)}=14.88 \mathrm{~m} / \mathrm{s}
$$

\section{c) Cálculo de tubería a presión:}

$$
\begin{aligned}
& \begin{aligned}
d=0.3 \sqrt[5]{\frac{Q^{2} L}{h}}=0.3\left[\frac{\left(0.0089 \frac{\mathrm{m}^{3}}{\mathrm{~s}}\right)^{2}(2 \mathrm{~m})}{12}\right]^{\frac{1}{5}} \\
=0.032 \mathrm{~m} \\
=31.71 \mathrm{~mm}(\text { Espesor } \text { pared })
\end{aligned} \\
& \begin{aligned}
h=\text { Altura del salto }(y) \\
L=\text { Longitud de la tuberia a presion }
\end{aligned}
\end{aligned}
$$

(x)

- $\quad$ Diámetro Interior $=101.0 \mathrm{~mm}$

- $\quad$ Diámetro Exterior $=107.1 \mathrm{~mm}$

- Diámetro Nominal=4”

\section{Determinación de rugosidad:}

Rugosidad absoluta: $\mathrm{k}=0.003 \mathrm{~mm}^{2}$

$$
\text { Relación }=\frac{k}{\text { diámetro int. de la tuberia }}=\frac{0.003 \mathrm{~mm}^{2}}{101.0 \mathrm{~mm}}=0.000030 \mathrm{~mm}
$$


d) Cálculo de velocidad en la tubería:

$$
v=\frac{4 Q}{\pi(\text { diámetro int. })^{2}}=\frac{4\left(0.0089 \frac{\mathrm{m}^{3}}{\mathrm{~s}}\right)}{\pi(0.101 \mathrm{~m})^{2}}=1.11 \mathrm{~m} / \mathrm{s}
$$

\section{Cálculo de Número de Reynolds:}

$R e=\frac{\text { Velocidad agua en la tubería } x \text { d.int. }}{\text { Viscosidad cinemática del agua } 20^{\circ} \mathrm{C}}=\frac{\left(1.11 \frac{\mathrm{m}}{\mathrm{s}}\right)(0.101 \mathrm{~m})}{1.004 \times 10^{-6} \frac{\mathrm{m}^{2}}{\mathrm{~s}}}=1.12 \times 10^{5}$

Rugosidad F: se calcula en base en el diagrama de Moody (Figura 2).

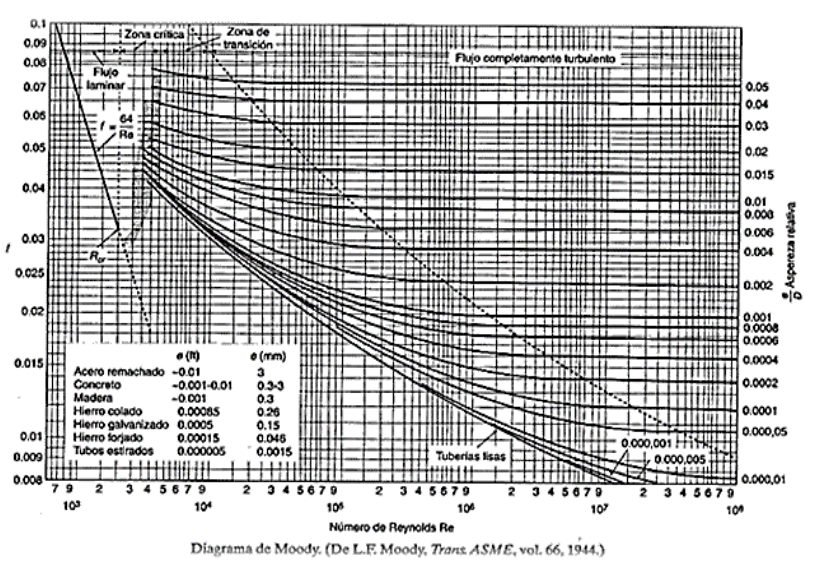

Figura 2 Diagrama de Moody

Fuente: Mecánica de fluidos. H. Shames Irving, $3^{a}$. Edición, McGraw-Hill. 1995

$\frac{k}{\text { d.interior }}=0.00003 \mathrm{mRe}=1.12 \times 10^{5}$

$f=0.029$

e) Cálculo de pérdida de carga hidráulica debido a fricción

Con base a la ecuación de Darcy Weisbach, la cual relaciona la pérdida de carga principal debido a la fricción del fluido a lo largo de una longitud determinada de tubería con la velocidad promedio (Shames, 316AD):

$h f=f \times \frac{L}{d} \times \frac{v^{2}}{2 g}=0.029 \times \frac{2 \mathrm{~m}}{0.101 \mathrm{~m}} \times \frac{(1.11 \mathrm{~m} / \mathrm{s})^{2}}{2\left(9.81 \frac{\mathrm{m}}{\mathrm{s}^{2}}\right)}=0.036 \mathrm{~m}$

En la tubería de PVC se pierde $0.036 \mathrm{~m}$ de carga hidráulica.

$h N=h-h f=12-0.036=11.964 m$

\section{f) Diseño de turbina}

\section{Potencia generada por turbina:}

$P=p g h N Q n$ $\mathrm{n}=$ eficiencia

hN= Carga hidráulica neta

$p=$ densidad

$\left.P=\left(100 \frac{\mathrm{kg}}{\mathrm{m}^{3}}\right)\left(9.81 \frac{\mathrm{m}}{\mathrm{s}^{2}}\right)(11.964 \mathrm{~m}) 0.0089 \frac{\mathrm{m}^{3}}{\mathrm{~s}}\right)(0.95)=992.33 \mathrm{w} \approx 1 \mathrm{~kW}$

Diámetro del chorro de la salida de la tobera:

$d o=\frac{\sqrt{4 Q}}{\pi k c \sqrt{2 g h N}}$

$\mathrm{Kc}=$ coeficiente de velocidad de tobera $\mathrm{o}$ chorro, entre 0.97 y 0.98 , en este caso se consideró 0.97 :

$d o=\frac{\sqrt{4\left(0.0089 \frac{\mathrm{m}^{3}}{\mathrm{~s}}\right)}}{\pi(0.97) \sqrt{2(9.81)(11.964 \mathrm{~m})}}=\frac{\sqrt{0.0356}}{46.68}=0.0276 \mathrm{~m}$

$\mathrm{d}=0.0276 \mathrm{~m}=2.76 \mathrm{~cm}=3 \mathrm{~cm}$

\section{Velocidad de salida:}

$V=\frac{4 Q}{\pi d o^{2}}=\frac{4\left(0.0089 \frac{\mathrm{m}^{3}}{\mathrm{~s}}\right)}{\pi(0.0276 \mathrm{~m})^{2}}=14.87 \mathrm{~m} / \mathrm{s}$

g) Determinación de Dimensiones de álabes de la cuchara:

\section{Diámetro del rodete:}

$D=\frac{k u \sqrt{2 g h n}}{\pi n}$

$\mathrm{ku}=$ Coeficiente de velocidad tangencial, varía entre 0.44 y 0.48 , consideramos 0.46 .

Número de revoluciones específico:

$n=\frac{60 f}{z}$

$f=$ Frecuencia en $\mathrm{Hz}$ consideramos $1350 \mathrm{rpm}$

$D=\frac{0.46 \sqrt{2\left(9.81 \mathrm{~m} / \mathrm{s}^{2}\right)(11.964)}}{\pi(1350 \mathrm{rpm})} \times 60=99.70 \mathrm{~mm}$

\section{Número de álabes de la turbina:}

Diferencia entre el radio del rodete y diámetro máximo, cresta de la cuchara. 
Da = Diámetro máx.cresta de la cuchara

$D a=D+2 d o$

$D a=99.70 \mathrm{~mm}+2(27.6 \mathrm{~mm})=154.9 \mathrm{~mm}$

$\lambda=\frac{D a-D}{2}$

$\lambda=\frac{154.9 \mathrm{~mm}-99.70 \mathrm{~mm}}{2}=27.6 \mathrm{~mm}$

\section{Relación entre diámetros:}

$\frac{\lambda}{d o}=\frac{27.6 \mathrm{~mm}}{27.6 \mathrm{~mm}}=1$

Ángulo comprendido entre el centro del rodete y el punto máximo de la salida del chorro:

$\varphi=\cos ^{-1}\left(\frac{1+\delta}{1+2 \mathrm{k} \delta}\right)$

$\delta=$ Relación entre el diámetro del chorro y el diámetro del rodete

$\delta=\frac{d o}{D}=\frac{27.6 \mathrm{~mm}}{99.70 \mathrm{~mm}}=0.276$

$\varphi=\cos ^{-1}\left(\frac{1+0.276}{1+(2 \times 1 \times 0.276)}\right)=0.822$

$$
=34.69
$$

Ángulo entre la arista de la cuchara y el punto máximo de la salida del chorro.

$\psi=2 \frac{k u}{k c} \sqrt{(1+2 k \delta)^{2}-(1+\delta)^{2}}$

$\psi=2\left(\frac{0.46}{0.97}\right) \sqrt{\left(1+2(1 \times 0.276)^{2}-(1+0.276)^{2}\right.}=0.837$

Valor paso máximo

\section{Ángulo existente entre cucharas:}

$\theta=2 \varphi-\psi=2(0.822)-(0.837)=0.807$

$\theta=0.807\left(\frac{180}{\pi}\right)=46.23^{\circ}$

\section{Número de cucharas:}

$z=\frac{2 \pi}{\theta}=\frac{2 \pi}{0.807}=8$ Paletas

\section{Resumen de los cálculos realizados:}

Caudal de bajada: 8.94 1/s.

Diámetro nominal de tubería antes de turbina: 4".

Velocidad de flujo en tubería: $1.11 \mathrm{~m} / \mathrm{s}$

Potencia de turbina: $1 \mathrm{~kW}$.

Diámetro nominal de chorro a la salida de turbina $1 \frac{1}{2}, "$

- $\quad$ Velocidad a la salida de la turbina 14.87 $\mathrm{m} / \mathrm{s}$.

Consideraciones principales: Temperatura ambiente del agua $20{ }^{\circ} \mathrm{C}$ y densidad de $1000 \mathrm{~kg} / \mathrm{m}^{3}$.

\section{Bomba:}

Dentro del tanque subterráneo en el nivel 2 se localiza una bomba sumergible que elevará el agua hasta el tanque 1 , cuyas especificaciones son:

$-1 / 2 \mathrm{hp}$ de potencia.

- Tubería de 1"

\section{Diseño del sistema}

El estanque se localiza en el nivel más bajo del terreno. Mantiene dimensiones de 2x3 m y una profundidad de $40 \mathrm{~cm}$, se considera un volumen de 1800 l. El agua pasa a través de una cascada artificial que permitirá un filtrado de partículas sólidas y un mejor diseño, además se colocó vegetación a un costado del estanque por lo que también se aprovechará el agua para riego.

El exceso se recirculará nuevamente al tinaco colocado en el techo del edificio a $12 \mathrm{~m}$ sobre el suelo. Se colocó una malla antes de que el agua ingrese al tinaco la cual detendrá las partículas o peces (figura 3 y 4 ).
CASTILLO-TÉLlEZ, Margarita, CASTILLO-TÉLlEZ, Beatriz, MEJÍA-PÉREZ, Gerardo Alberto y HERNÁNDEZCRUZ, Luz María. Aprovechamiento sustentable del recurso hídrico mediante un sistema de captación de agua de lluvia y red de suministro. Revista de Ingeniería Innovativa. 2020 


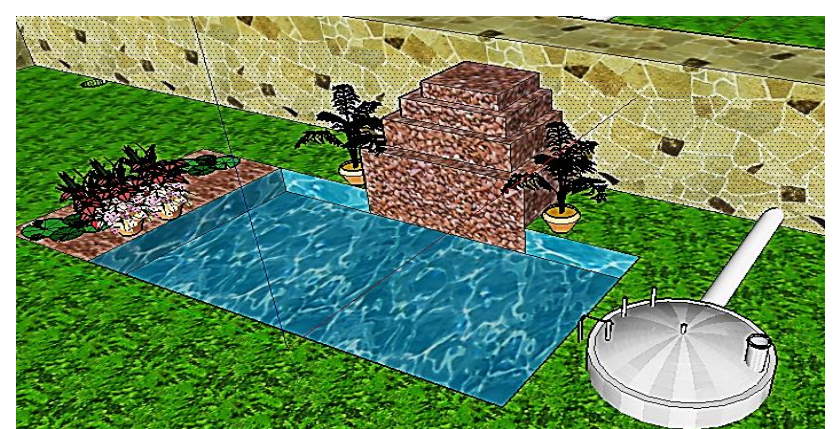

Figura 3 Modelado del sistema en la sección de la arquitectura del estanque con el programa Sketchup Fuente: Diseño de autor

El primer sistema de filtración se encuentra antes de bajar el agua por la tubería, para no dañar los álabes de la microturbina. Se aprovecha la energía potencial del agua.

El sistema tiene suministro de agua por medio de la red disponible y mediante la captación de agua de lluvia que entrará por la válvula check cuando el nivel de la azotea tienda a subir (figura 5). En la figura 6 se muestra la representación de la turbina. Se disecciona la tubería dirigiéndose hacia un destilador solar.

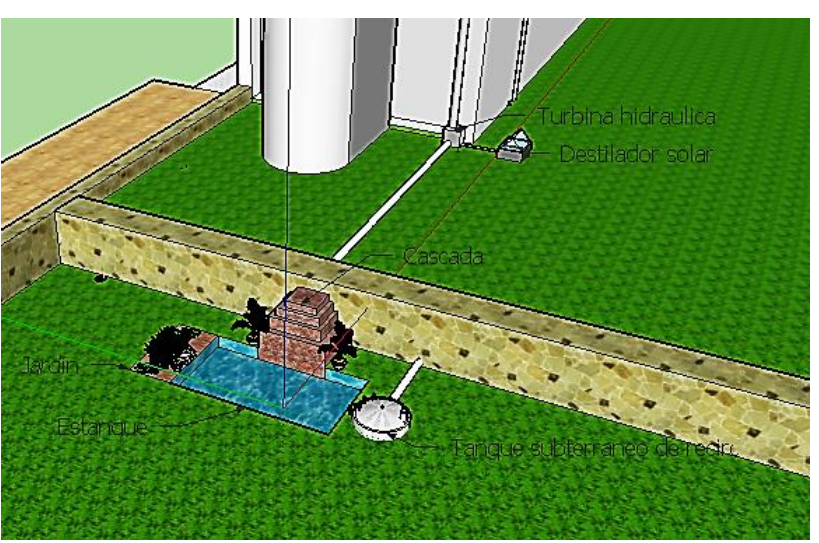

Figura 4 Vista lateral del estanque. Nivel 1 (sobre el suelo) y Nivel 2 (localización del sistema)

Fuente: Diseño de autor

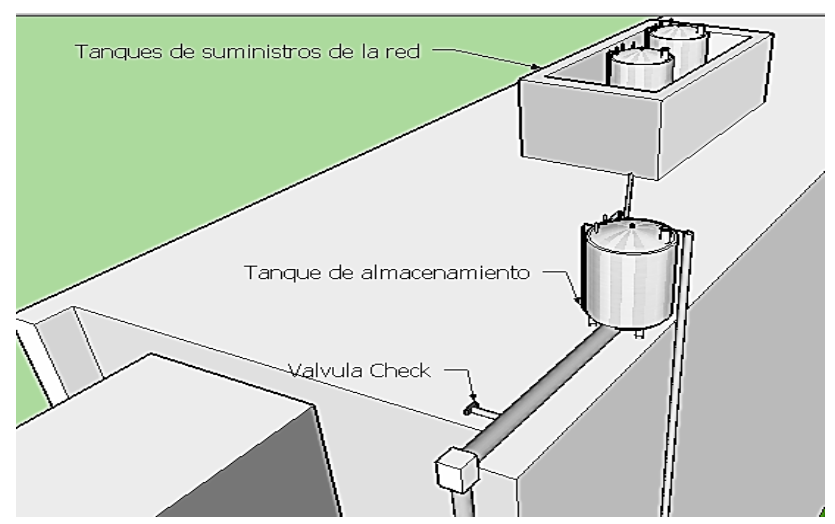

Figura 5 Vista superior del tanque sobre el edificio B Fuente: Diseño de autor

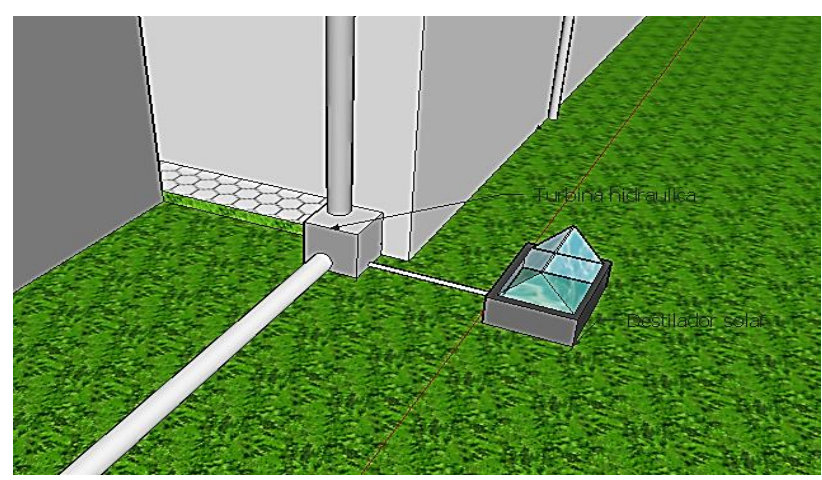

Figura 6 Ubicación de la turbina en el nivel 1 y disección de la tubería hacia un destilador solar Fuente: Diseño de autor

En la figura 7 se muestra el plano arquitectónico principal del sistema de captación de agua de lluvia diseñado con el programa Autocad.

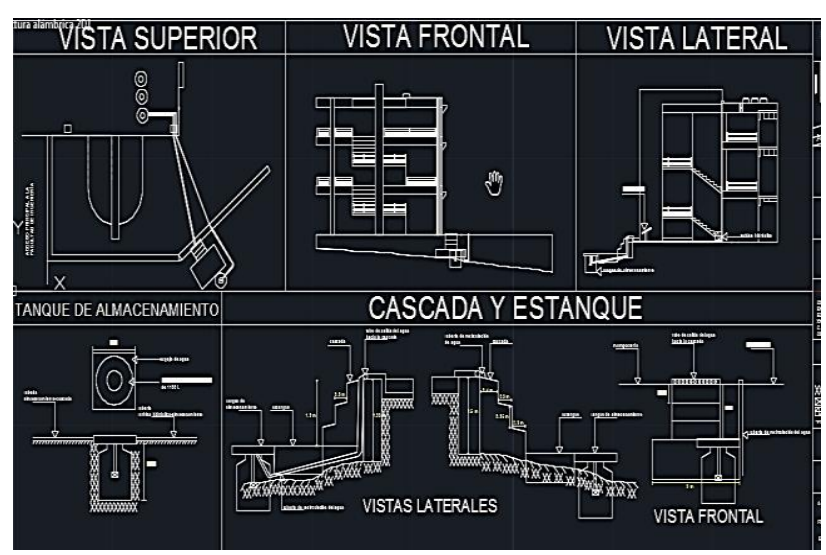

Figura 7 Vistas del edificio B y colocación del sistema de almacenamiento

Fuente: Diseño de autor

Para el área de alimentación de los destiladores solares, se consideró una tubería de $1 / 2$ " ya que se requiere un caudal muy pequeño para realizar la destilación. La válvula check introduce agua al sistema cuando llueve. Al subir el nivel en la azotea permite acceso del agua y no se requiere tomar agua de la red (los tinacos). Se contempla una válvula para cerrar el paso del agua entre los tinacos de suministro y el tinaco del sistema. En la figura 8 se presenta el sistema de captación de agua durante diferentes etapas de construcción.
CASTILLO-TÉLlEZ, Margarita, CASTILLO-TÉLLEZ, Beatriz, MEJÍA-PÉREZ, Gerardo Alberto y HERNÁNDEZCRUZ, Luz María. Aprovechamiento sustentable del recurso hídrico mediante un sistema de captación de agua de lluvia y red de suministro. Revista de Ingeniería Innovativa. 2020 


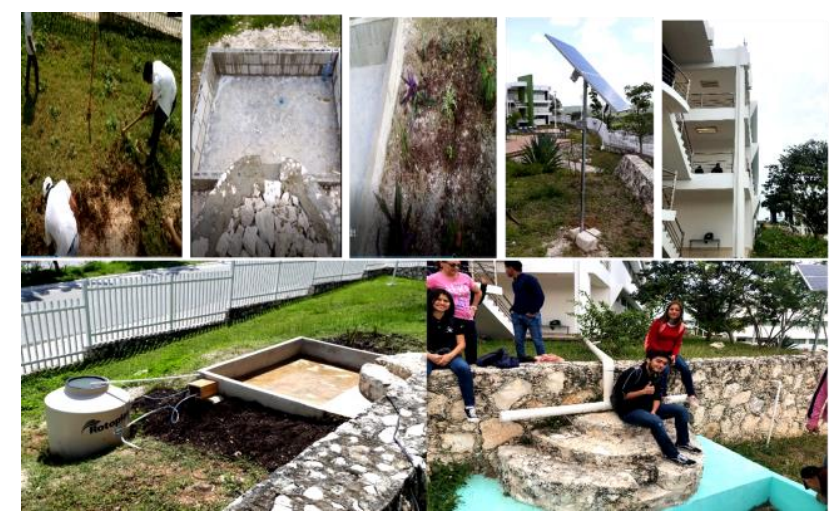

Figura 8 Construcción e implementación del sistema de captación de agua en sus diferentes etapas.

Fuente: Fotos de autor

\section{Agradecimientos}

A todos los alumnos de Ingeniería en Energía que participaron en la construcción del sistema de captación de agua de lluvia. Especialmente a Edwin Álvarez Caamal, Ricardo Isasi Ábrego y Jonathan Castillo Estrella, quienes liderearon la participación de alumnos.

\section{Conclusiones}

De manera general el proyecto representa una aplicación de la energía potencial que puede ser aprovechada desde una altura determinada, así como los diferentes usos que se puede dar al recurso hídrico btenido de la captación de agua de lluvia. Este sistema funciona de forma óptima y constituye una herramienta importante como complemento a los diferentes programas de estudio de la carrera de Ingeniería en Energía, permitiendo a los alumnos estudiar cada uno de sus componentes en condiciones reales de operación.

El agua, como recurso estratégico, depende del manejo sostenible de los ecosistemas y por esta razón debe ser una prioridad nacional y mundial, en la actualidad es de gran importancia promover estrategias que apoyen a que la población adquiera el hábito del aprovechamiento del recurso hídrico mediante los diferentes métodos de captación de agua de lluvia, los cuales son sencillos, de nulo mantenimiento y por lo tanto de fácil adopción.

\section{Referencias}

Anaya, M. (2011). Captación del agua de lluvia. Solución caída del cielo, 132.

CONAGUA. (2018). Estadísticas del agua en México 2018 (p. 368).
En, C., \& Nivel, C. D. E. (2000). Agricultura de humedad residual a través de surcos y camellones en curvas de nivel, brasil, 187-194.

FAO. (2013). Captación Y Almacenamiento De Agua De Lluvia. Santiago de Chile. https://doi.org/10.1111/jce.13019

Guía De Diseño Para Captación Del Agua De Lluvia. (2004).

López, N. A., Palacios, O. L., Anaya, M., Chávez, J., \& Enrique, J. (2017). Diseño de sistemas de captación del agua de lluvia: alternativa de abastecimiento hídrico * Rainwater harvesting systems design: an alternative for water supply Resumen Introducción. Revista Mexicana de Ciencias Agrícolas, 8, 1433-1439.

Robles, M., Näslund-hadley, E., Ramos, M. C., \& Paredes, J. R. (2015). Manejo Sostenible del Agua.

Shames, I. H. (316AD). Mecánica de fluidos.
CASTILLO-TÉLlEZ, Margarita， CASTILLO-TÉLlEZ, Beatriz, MEJÍA-PÉREZ, Gerardo Alberto y HERNÁNDEZCRUZ, Luz María. Aprovechamiento sustentable del recurso hídrico mediante un sistema de captación de agua de lluvia y red de suministro. Revista de Ingeniería Innovativa. 2020 